$\Omega_{1}$ ARTIGO DE REVISÃO

1 Granted Projects, SonaeMC,

Rua João Mendonça

ก. ${ }^{\circ} 529$,

4464-501 Senhora da

Hora, Matosinhos, Portugal

2 Direção da Qualidade e Investigação, Sonae MC

Estrada da O

118,

Parque Holanda - Bloco D,

2790-114 Carnaxide,

Portugal

Endereço para correspondência

Daniela Correia

Rua Palmares, Lote 6,

2680-159 Camarate, Loures,

Portugal

daniela.correia.lcna@gmail.com

Histórico do artigo

Recebido a 5 de novembro

de 2020

Aceite a 29 de março de 2021

\title{
SUCRALOSE: AMIGA OU INIMIGA?
}

\author{
SUCRALOSE: FRIEND OR ENEMY?
}

Daniela Correia"; Mayumi Thaís Delgado²; Sofia Costa Dinis²

RESUMO

O uso de edulcorantes é uma estratégia para a diminuição do consumo de açúcar. A sucralose é dos mais utilizados, sendo descrita como segura por diferentes entidades de referência, como a Autoridade Europeia para a Segurança Alimentar e a Food \& Drug Administration. Mas sendo as publicações por tais entidades anteriores a 2017, torna-se importante atualizar o conhecimento científico. Foi feita uma pesquisa na base de dados Pubmed com o termo "sucralose AND health", para os últimos 5 anos. Os principais temas abordados são a relação entre a ingestão de sucralose e a gestão da glicémia, ganho de peso, risco de desenvolvimento de síndrome metabólica e alteração da microbiota intestinal, com resultados, em geral, inconclusivos. Surgem estudos que levantam preocupações sobre exposição no útero e possível insegurança da sucralose, ainda pouco representativos. Contudo, perante a evidência atual, não é possível aferir que a sucralose leve a efeitos negativos na saúde, quando respeitado a ingestão diária aceitável (ADI, Acceptable Daily Intake).

\section{PALAVRAS-CHAVE}

Edulcorantes, Saúde, Segurança Alimentar, Sucralose

\section{ABSTRACT}

The use of sweeteners is a strategy for reducing sugar consumption. Sucralose is one of the most used, being described as safe by different reference entities, such as European Food Safety Authority and Food \& Drug Administration. But since the publications by these entities are prior to 2017, it is important to update scientific knowledge. A search was made in the Pubmed database with the term "sucralose AND health", for the last 5 years. The main topics addressed are routes associated with blood glucose management, weight gain, metabolic syndrome and intestinal microbiota, with results in general inconclusive. Studies are emerging that raise concerns about exposure in the uterus and possible insecurity of sucralose. Even so, based on current evidence, it's not possible to assess that sucralose leads to negative health effects, when the ADI is respected. But its use/consumption should be moderate, since the long-term effects are not fully known.

KEYWORDS

Sweeteners, Health, Food Safety, Sucralose

\section{INTRODUÇÃO}

Os efeitos negativos da ingestão excessiva de açúcar na saúde são amplamente conhecidos e muitas são as estratégias para a diminuição do seu consumo, desde a educação alimentar às intervenções políticas. O uso de edulcorantes em produtos alimentares tem sido adotado como uma alternativa ao açúcar (1). Estes são aditivos definidos pela legislação como substâncias usadas para conferir um sabor doce aos alimentos ou em adoçantes de mesa (2). Podem ser de duas categorias: edulcorantes de alta intensidade, substâncias com um sabor doce intenso e sem valor energético, e polióis, definidos como "álcoois contendo mais de dois grupos hidroxilo, que fornecem poucas calorias, mas também podem ser usados para outros fins que não edulcorantes" (3). De entre os primeiros, a sucralose (E995) é dos mais utilizados, com capacidade adoçante 600 vezes superior ao açúcar (4). Em 1991, o Comité Conjunto de Especialistas em Aditivos Alimentares da Organização das Nações Unidas para a Alimentação e a Agricultura/Organização Mundial da Saúde estabeleceu uma ingestão diária aceitável (ADI) de 0-15 mg/kg peso corporal/dia (mg/Kg pc/d) (5). O seu uso encontra-se aprovado por autoridades em cerca de 80 países (6), por exemplo, pelo Comité Científico da Comissão Europeia para a Alimentação, que refere existirem evidências da segurança do seu uso (7), e pela Food \& Drug Administration (FDA), que aprovou um ADI de $5 \mathrm{mg} / \mathrm{kg}$ pc/d $(8,9)$. Contudo, as preocupações da população e da comunidade científica mantêm-se. Em 2016, foi publicado um estudo que relatou um aumento da incidência de tumores malignos e neoplasias hematopoiéticas em ratos, associados ao consumo de 
sucralose (10). Mas a Autoridade Europeia para a Segurança Alimentar (EFSA) publicou um parecer onde critica o desenho do estudo e as suas práticas, referindo ainda que os dados disponíveis não apoiavam as conclusões do ensaio (11). Apesar das seguranças dadas por entidades de referência, o consumidor mantém as suas preocupações, possivelmente em parte potenciadas por outras fontes de informação não fidedigna que partilham artigos que relatam apenas os efeitos negativos do consumo de sucralose na saúde. Por outro lado, reparamos que publicações de entidades de referência são anteriores a 2017, inclusive, e que muitos trabalhos focam os edulcorantes como um todo (3). Sendo a sucralose amplamente utilizada, torna-se importante atualizar o conhecimento científico relativamente aos efeitos do seu consumo. Para tal fez-se uma pesquisa bibliográfica na base de dados Pubmed com o termo "Sucralose AND health", com filtro para o período 2015-2020, com última atualização a 7 de agosto de 2020, tendo sido resultados da pesquisa um total de 117 artigos. Excluíram-se os artigos que não tratam tópicos referentes à segurança da sucralose e/ou a influência na saúde, e os que tratam edulcorantes no geral, sem especificar a sucralose. Não se incluiu a publicação relativa ao desenvolvimento de tumores em ratos (10), previamente analisada e refutada pela EFSA (11). Os artigos utilizados, perfazendo um total de 39 artigos, foram agrupados e tratados por tema.

\section{Regulação da Glicose e Insulina}

Os efeitos da sucralose na gestão da glicemia continuam a ser muito estudados. Um estudo de 2017, ex vivo, relatou que a administração intra-luminal no intestino delgado de ratos não afetou a secreção de incretinas, mas que a administração intra-arterial (10 mM) levou ao aumento de GLP-1 (12). Um estudo de 2019 com humanos avaliou o efeito de exposição oral à sucralose (sham feeding - consistia em bochechar sucralose sem ingerir) versus ingestão, usando $48 \mathrm{mg}$ de sucralose diluída em água (valor abaixo do ADI). Em indivíduos normoponderais, verificou-se diminuição da insulina plasmática com a exposição e a ingestão, e aumento da sensibilidade à insulina, em cerca de 52\%, com ingestão. Em obesos, a ingestão de sucralose levou ao aumento da insulina plasmática, mas a exposição levou ao decréscimo, e nenhuma intervenção afetou a sensibilidade à insulina. Em ambos os grupos, a ingestão de sucralose aumentou a área incremental sob a curva da resposta glicémica em cerca de $30 \%$ (13). Outro estudo com humanos, de 2020, demonstrou que o consumo diário de 136 mg de sucralose, diluída em água, não leva a alterações dos níveis de glicose, insulina, GLP-1 ou leptina no sangue (14). No entanto, o estudo incluiu uma amostra pequena $(n=17)$ e decorreu durante apenas 2 semanas. Uma revisão de 2017 concluiu que ensaios clínicos em humanos, coletivamente, não indicam efeitos adversos da sucralose no controlo da glicemia, peptídeo C, insulina, hemoglobina glicada, hormonas intestinais ou função intestinal (15). Outra revisão de 2017 refere que a maioria da evidência aponta para um efeito nulo na secreção de moléculas reguladoras do metabolismo da glicose. Refere ainda não existir evidência de efeito na absorção de glicose em humanos, e resultados contraditórios sobre efeitos na insulina ou tolerância à glicose (16). Em 2018, os autores de uma meta-análise concluíram que, apesar das diferenças estruturais entre os edulcorantes estudados (aspartame, sacarina, stevia e sucralose), o seu consumo não exerce efeitos sobre a glicemia (17). Numa revisão de 2019, de três ensaios clínicos randomizados sobre o efeito da sucralose, um revelou não existirem efeitos na glicemia, enquanto os outros dois obtiveram dados concordantes de diminuição da sensibilidade à insulina, mas contraditórios sobre a resposta aguda de insulina (18). Estes estudos usaram doses realistas (36 mg (juntamente com dextrose e maltodextrina) e $200 \mathrm{mg}$ ), mas uma curta duração (2 e 4 semanas). Os autores da revisão concluem que a evidência não é suficiente para aferir efeitos do uso de sucralose (18). Uma revisão de ensaios clínicos de 1969-2019 refere não ser possível concluir que o consumo de sucralose tenha efeitos no metabolismo da glicose e nas hormonas intestinais. Revela também que a maioria da evidência aponta para um efeito neutro sobre a glicemia, e que existem poucos estudos sobre os efeitos na sensibilidade à insulina (3 relatam diminuição e 1 reporta aumento) e GLP-1 (4 relatam aumento) (19).

\section{Aumento de Peso e Síndrome Metabólico}

Um estudo de 2019 observou que, em concentrações superiores ou iguais a $5 \mathrm{mM}$, a sucralose induziu a expressão de genes de stress no retículo endoplasmático em células hipotalâmicas de ratos, 0 que tem sido associado ao desenvolvimento de resistência central à leptina. Em concentrações superiores ou iguais a $10 \mathrm{mM}$, a sucralose apresentou ação citotóxica sobre as células (20). Um estudo de 2020, com células de estroma mesenquimais em meio adipogénico, observou uma regulação positiva de genes associados ao aumento da gordura intracelular (concentrações de 0,45e $1 \mathrm{mM}$ ) e aumento de acumulação de radicais de oxigénio (1 mM). Concentrações de 0,2 e 1 mM diminuíram a viabilidade das células (21). Um estudo de 2018 verificou que ratos suplementados com $1 \mathrm{~g}$ de adoçante comercial (12 mg de sucralose associada a maltodextrina), via água, tiveram menor aumento de peso inicial, mas acabaram com um peso significativamente superior (22). Outro estudo, também em ratos, verificou que apesar de não existirem diferenças na ingestão calórica entre grupos, o consumo de sucralose levou a um peso corporal inferior em ratos com dieta normal, mas não com uma dieta rica em gordura (HF). No entanto, verificaram-se consumos de sucralose excessivos, de $\sim 3300$ e $\sim 1500 \mathrm{mg} / \mathrm{kg}$ pc/d, no grupo de dieta normal e HF, respetivamente (23). Em humanos, um estudo de 2018 não encontrou associação entre o Síndrome Metabólico e o consumo de edulcorantes, mas observou uma associação positiva entre a redução da circunferência da cintura e o consumo total de edulcorantes, de sacarina e de sucralose (24). No entanto, os dados sobre consumo assentam na memória dos participantes (3 registos $24 \mathrm{~h}$ anteriores). Para além disso, o consumo de edulcorantes poderia ter sido iniciado numa tentativa de alterar a sua forma física/perder peso, o que não foi discutido.

\section{Microbiota e Ploblemas Gastrointestinais}

Outro tópico muito estudado é a influência da sucralose ao nível da microbiota intestinal. Dois estudos de 2018 usaram modelos de E. coli, e relataram um efeito bacteriostático da sucralose $(23,25)$. As concentrações de 1 e $100 \mathrm{mg} / \mathrm{ml}$ causaram citotoxicidade e genotoxicidade, respetivamente, em estirpes modificadas para detetar estes efeitos (25). Já em ratos (consumo $\sim 3300$ e $\sim 1500$ mg/kg pc/d nos grupos de dieta normal e HF, respetivamente, via água) observou-se que, não havendo diferenças no comportamento alimentar entre grupos, em ratos alimentados com uma dieta normal, a sucralose aumentou o filo Firmicutes e o genéro Bifidobacterium, enquanto que com uma dieta HF observou-se um efeito apenas nos Firmicutes, aumento que se associa à obesidade (23). No entanto, como mencionado anteriormente, a quantidade diária de sucralose consumida ao longo do estudo foi bastante elevada. Uma revisão de 2019, que analisa os edulcorantes de uma forma geral e os seus efeitos na microbiota intestinal, salienta 2 estudos em ratos sobre sucralose que relatam alterações da microbiota intestinal (26). Um dos estudos acompanha a administração de um 
adoçante comercial em doses correspondendo a 1,1-11 mg de sucralose/kg pc/d, diluída em água, por alimentação forçada, durante 12 semanas. Observou-se uma diminuição de bactérias intestinais benéficas, aumento do $\mathrm{pH}$ fecal, mudanças histopatológicas no cólon e aumento da expressão intestinal de P-gp, CYP3A4 e CYP2D1, conhecidos por reduzirem a biodisponibilidade de medicamentos administrados por via oral (26). O último estudo, e mais recente, conclui que o consumo de sucralose durante 6 meses, numa concentração alinhada com a ADI (não mencionada de forma concreta), pode afetar a microbiota intestinal e a sua dinâmica de desenvolvimento, levando a um aumento do risco de desenvolvimento de inflamação $(26,27)$. Um estudo de 2018, também com adoçante comercial (sucralose e maltodextria, $1: 99 \mathrm{w} / \mathrm{w}$ ), administrado via água a ratos (modelo normal e modelo espontâneo com ileíte semelhante a doença de Crohn - SAMP) durante 6 semanas, observou que o adoçante não aumentou a gravidade da ileíte, mas aumentou a atividade de mieloperoxidase em ratos SAMP, o que pode exacerbar o estado inflamatório. Promoveu ainda disbiose, com expansão das proteobactérias em ambos os modelos, proliferação excessiva de E. coli e aumento da infiltração bacteriana na lâmina própria ileal de ratos SAMP (28). Contudo, não é apresentada a dose de sucralose consumida e foram criticadas e discutidas algumas práticas deste estudo, pondo em causa a sua fiabilidade (29). Um outro estudo, de 2019, trabalhou amostras fecais de humanos saudáveis. Observou-se que a sucralose (50\% do ADI, sem referência se 5 ou 15 mg/kg pc/d) levou a um aumento da produção de ácido valérico, 5,6\% de variância na estrutura da comunidade microbiana, com aumento da abundância relativa de Escherichia/Shigella e de Bilophila, alterações que podem associar-se a inflamação (30). Duas revisões recentes, 2019 e 2020 , discutem que mais de $85 \%$ da sucralose consumida chega ao cólon, no entanto não, existem evidências de metabolização por bactérias intestinais, pelo que alterações no microbiota intestinal devem ser explicadas por mecanismos alternativos, como mudanças na dieta, doses exageradas, e/ou coadministração de outras substâncias (31, 32) o que normalmente não é discutido nos artigos.

\section{Outras Patologias}

Um estudo de 2019, em ratos, demonstrou através da análise proteómica que a sucralose (em água, durante 6 meses) desregula o metabolismo hepático proteico. Esta regulação negativa pode levar à inativação ribossomal no fígado, associada a inflamação mediada por citocinas (33). Por outro lado, com base num estudo realizado em humanos, a sucralose pode ter aplicação terapêutica em casos de hipotensão pós-prandial, que afetam sobretudo idosos e diabéticos, uma vez que não leva a mudanças na pressão arterial ou no fluxo sanguíneo da artéria mesentérica superior, ao contrário do que acontece com a glicose (34).

\section{Mãe, Bebé e Infância}

Existem alguns estudos sobre o efeito do consumo de sucralose durante a gravidez, na descendência. Num estudo em ratos de 2020, observou-se que a exposição de mães a sucralose (em água, com uma ingestão de 5-15 mg/kg/d) pode alterar a composição e diversidade da microbiota intestinal, reduzir o número de bactérias produtoras de butirato, interferir no desenvolvimento intestinal, induzir a inflamação de baixo grau e interromper a função de barreira intestinal nos descendentes com 3 semanas de idade, podendo ainda contribuir para a suscetibilidade à esteatose hepática, em adultos (35). Outro artigo de 2020, analisou dados relativos a células, ratos e humanos. Em adipócitos, a exposição à sucralose (200nM) em estágios iniciais de diferenciação aumentou a acumulação de lipídios e expressão de genes de diferenciação, o que também se verificou in vivo. Em ratos, o consumo de $6,3 \mathrm{mg} / \mathrm{kg}$ pc/d de sucralose durante a gravidez levou a maior peso, maior adiposidade e resistência à insulina nas crias, especialmente em machos. Em humanos, com base num estudo retrospetivo, observou-se que crianças filhas de mães que consumiam regularmente bebidas com edulcorantes durante a gravidez, aos 3 anos de idade, tinham maior índice de massa corporal e maior grau de adiposidade (36). Um estudo de 2018, observou que após o consumo de um refrigerante diet ( $\sim 355 \mathrm{ml}$, com $68 \mathrm{mg}$ de sucralose e $41 \mathrm{mg}$ de acessulfame-K) encontra-se sucralose no leite materno humano (pico médio de 8,1 $\mathrm{ng} / \mathrm{mL}$ ). A ingestão deste leite poderá levar aos problemas discutidos causados pelo consumo de sucralose, podendo também modelar as preferências do bebé pelo sabor doce $(37,38)$.

Nas crianças, a assimilação da sucralose é semelhante à dos adultos, no entanto é de notar que por terem uma dimensão inferior, ao consumirem uma dose destinada a um adulto, terão uma concentração sanguínea de sucralose superior à encontrada no adulto (39). No entanto, dados de exposição sugerem que crianças e adolescentes, não ultrapassam a ADI de $15 \mathrm{mg} / \mathrm{kg}$ pc/d (40, 41, 42), mesmo as com patologias como Diabetes Mellitus tipo 1 (43), fenilcetonuria $(44,45)$ ou alergia severa à proteína do leite $(45)$.

\section{Segurança}

Em linha com a opinião de entidades de referência como a EFSA e FDA, uma extensa revisão de 2017, após analisar artigos sobre toxicocinética e genotoxicidade em animais e humanos, concluiu que a sucralose é segura (15). Porém, surgem novos estudos que revelam o contrário. Em 2018, um estudo com ratos, identificou dois novos produtos de biotransformação (formas acetiladas da sucralose, mais lipofílicas), detetados na urina e fezes, durante o período de dosagem, e na urina até 11 dias após a interrupção do consumo de sucralose. Estes metabolitos foram ainda encontrados no tecido adiposo 2 semanas após a interrupção da administração de sucralose,

\section{Figura 1}

Infografia resumo das principais conclusões do estudo de revisão relativo à segurança para a saúde humana do consumo do edulcorante sucralose (12-50)

EDULGORANTES COMO ALTERNATIVA AO AÇÚGAR: SUCRALOSE - AMIGA OU INIMIGA?

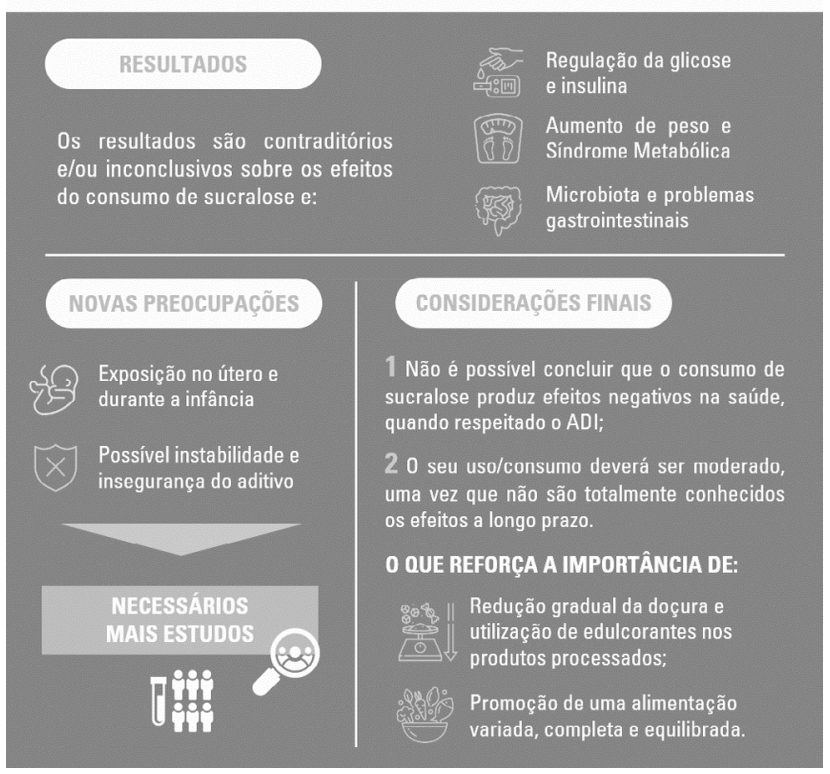


o que poderá levar à proliferação de pré-adipócitos. Os autores referem que um fator de segurança de 100 vezes foi aplicado aos dados de toxicidade da sucralose para estabelecer o ADI, mas uma vez que se observou a bioacumulação de sucralose no tecido adiposo, tal facto justificaria a redução do ADI para $<1 \mathrm{mg} / \mathrm{kg}$ pc/d (46). No entanto, tal deverá ser averiguado com maior profundidade. Um estudo de 2020, que utilizou cultura de linfócitos humanos e concentrações de sucralose abaixo do LC50 (1, 10, e $50 \mu \mathrm{g} / \mathrm{ml})$, observou que a sucralose reduz não seletivamente os linfócitos totais devido a quedas nos níveis de CD4+, CD8+ e CD4+ CD8+, entre 25 e 81\%. Observou ainda um aumento nos danos ao DNA e mudanças estruturais nos conjuntos cromossómicos (47). Num estudo de 2020, que usou um modelo de ratos para estudar a indução de cancro colorretal, observou que o consumo de sucralose na água (sem indicação da dose total) causou aumentos significativos no número e tamanho dos tumores colorretais, alterando outros parâmetros, como peso corporal e do baço, $\beta$-glucuronidase fecal, proteases digestivas, microbiota intestinal, citocinas e vias inflamatórias, moléculas da via de sinalização de tumor STAT3/VEGF, scores patológicos e mortalidade (48).

As principais conclusões retiradas da pesquisa efetuada e considerações finais são adicionalmente apresentadas na Figura 1 , no formato de infografia.

\section{ANÁLISE CRÍTICA}

A literatura científica atual apresenta de uma forma geral resultados inconclusivos ou contraditórios. Tal pode dever-se ao uso de metodologias bastante variáveis. É ainda de salientar o facto de serem usados diferentes veículos de sucralose (água, refrigerantes, etc.), encontrando-se pouco estudada a influência da matriz alimentar. Da pesquisa efetuada, apenas 2 artigos discutem este tema, um referindo que a concentração sanguínea de sucralose é comparável após a ingestão de sucralose a partir de diferentes matrizes alimentares (39), e outro referindo que as respostas de GLP-1 são diferentes (49). Adicionalmente, observam-se limitações recorrentes(15): I. Não descrição da fonte de sucralose (ausência total ou menção ao uso de adoçante comercial, cuja composição não é apresentada); II. Ausência da dose (por desconhecimento da formulação da fonte ou por não contabilização da ingestão, comum em ensaios com animais, com administração em água); III. Ignorada a coadministração da sucralose com outros edulcorantes (ex.: adoçantes comerciais e refrigerantes diet); IV. Não contabilização da ingestão alimentar, calórica e de água. Outro ponto a discutir é o facto de serem usadas doses/ concentrações pouco realistas. Em células, concentrações perto de 10 mM são citotóxicas, contudo, esta dose parece ser muito elevada, uma vez que o ADI de $15 \mathrm{mg} / \mathrm{kg}$ pc/d corresponderia a 0,11 mM/kg/d (20). Segundo outro estudo, no sistema circulatório de indivíduos que consomem sucralose em doses elevadas será plausível observar a concentração de 0,2 mM (21). São necessários estudos mais robustos para aumentar o conhecimento científico, especialmente estudos que utilizem doses mais baixas, uma vez que a literatura revela que a ingestão de sucralose está longe de ultrapassar o ADI estabelecido (50), mesmo em grupos considerados de risco, como as crianças, incluindo as que necessitam de produtos especializados (40-45).

\section{CONCLUSÕES}

A literatura atual revela resultados contraditórios e/ou inconclusivos sobre os efeitos do consumo de sucralose na saúde. Têm surgido preocupações relativas à exposição in utero e durante a infância e sobre a possível insegurança do aditivo, mas são necessários mais trabalhos nestas áreas. As principais conclusões retiradas da pesquisa efetuada e considerações finais são adicionalmente apresentadas na
Figura 1, no formato de infografia.

Perante a evidência atual, não é possível concluir que a sucralose seja causadora de efeitos negativos na saúde, quando respeitado o ADI. Contudo, o seu uso/consumo deverá ser moderado, uma vez que não são totalmente conhecidos os efeitos a longo prazo. A indústria poderá ter um papel importante ao reduzir o uso de aditivos reduzindo gradualmente o sabor doce dos alimentos processados. Outra estratégia poderá assentar na promoção de uma alimentação variada, já que permite reduzir a ingestão total de ingredientes e/ou aditivos específicos.

\section{REFERÊNCIAS BIBLIOGRÁFICAS}

1. World Health Organization, "Incentives and disincentives for reducing sugar in manufactured foods: An exploratory supply chain analysis.," 2017.

2. Europeu,P. Regulamento (CE) № 1333/2008 Parlamento Europeu e do Conselho relativo aos aditivos alimentares. Jornal Oficial da União Europeia. 2007, 16-33.

3. European Commission, "Sugars and Sweeteners," Last update: March 2020. [Online]. Available: https://ec.europa.eu/jrc/en/health-knowledge-gateway/promotionprevention/nutrition/sugars-sweeteners.

4. Sylvetsky AC, Rother KI. Trends in the consumption of low-calorie sweeteners. Physiol Behav. 2016;164(Pt B):446-450.

5. Joint FAOMHO Expert Committee on Food Additives. Evaluation of certain food additives and contaminants: thirty-seventh report of the Joint FAO/WHO Expert Committee on Food Additives. Meeting held in Geneva from 5 to 14 June $1990 \_1991$. 6. Kroger, M., Meister, K., \& Kava, R. Low-calorie sweeteners and other sugar substitutes: a review of the safety issues. Comprehensive reviews in food science and food safety. 2006; 5(2), 35-47.

7. European Commission Scientific Committee on Food. Opinion of the Scientific Committee on Food on sucralose. 2000.

8. US Food and Drug Administration. Food additives permitted for direct addition to food for human consumption; sucralose. Federal Register, 1998; 63(64), 16417-16433. 9. US Food and Drug Administration, "Food additives permitted for direct addition to food for human consumption; sucralose," 1999.

10. Soffritti, M., Padovani, M., Tibaldi, E. et. al. Sucralose administered in feed, beginning prenatally through lifespan, induces hematopoietic neoplasias in male swiss mice. Int J Occup Environ Health. 2016;22(1):7-17.

11. EFSA Panel on Food Additives and Nutrient Sources added to Food (ANS), et al., "Statement on the validity of the conclusions of a mouse carcinogenicity study on sucralose (E 955) performed by the Ramazzini Institute," EFSA Journal. 2017; 15(5). 12. Saltiel MY, Kuhre RE, Christiansen CB, et al. Sweet Taste Receptor Activation in the Gut Is of Limited Importance for Glucose-Stimulated GLP-1 and GIP Secretion. Nutrients. 2017;9(4):418.

13. Nichol AD, Salame C, Rother KI, Pepino MY. Effects of Sucralose Ingestion versus Sucralose Taste on Metabolic Responses to an Oral Glucose Tolerance Test in Participants with Normal Weight and Obesity: A Randomized Crossover Trial. Nutrients. 2019;12(1):29.

14. Ahmad SY, Friel JK, MacKay DS. The effect of the artificial sweeteners on glucose metabolism in healthy adults: a randomized, double-blinded, crossover clinical trial. Appl Physiol Nutr Metab. 2020;45(6):606-612.

15. Magnuson BA, Roberts A, Nestmann ER. Critical review of the current literature on the safety of sucralose. Food Chem Toxicol. 2017;106(Pt A):324-355.

16. Chan CB, Hashemi Z, Subhan FB. The impact of low and no-caloric sweeteners on glucose absorption, incretin secretion, and glucose tolerance. Appl Physiol Nutr Metab. 2017;42(8):793-801.

17. Nichol AD, Holle MJ, An R. Glycemic impact of non-nutritive sweeteners: a systematic review and meta-analysis of randomized controlled trials. Eur J Clin Nutr. 2018;72(6):796-804.

18. Ahmad SY, Azad MB, Friel J, MacKay D. Recent evidence for the effects of nonnutritive sweeteners on glycaemic control. Curr Opin Clin Nutr Metab Care. 2019;22(4):278-283. 
19. Ahmad SY, Friel JK, Mackay DS. Effect of sucralose and aspartame on glucose metabolism and gut hormones. Nutr Rev. 2020;78(9):725-746.

20. Park S, Sethi S, Bouret SG. Non-nutritive Sweeteners Induce Hypothalamic ER Stress Causing Abnormal Axon Outgrowth. Front Endocrinol. 2019;10:876.

21. Kundu N, Domingues CC, Patel J, et al. Sucralose promotes accumulation of reactive oxygen species (ROS) and adipogenesis in mesenchymal stromal cells. Stem Cell Res Ther. 2020;11(1):250.

22. Barrios-Correa AA, Estrada JA, Martel C, Olivier M, López-Santiago R, Contreras I. Chronic Intake of Commercial Sweeteners Induces Changes in Feeding Behavior and Signaling Pathways Related to the Control of Appetite in BALB/c Mice. Biomed Res Int. 2018;2018:3628121

23. Wang QP, Browman D, Herzog H, Neely GG. Non-nutritive sweeteners possess a bacteriostatic effect and alter gut microbiota in mice. PLoS One. 2018;13(7).

24. Hess, E. L., Myers, E. A., Swithers, S. E., \& Hedrick, V. E. Associations between nonnutritive sweetener intake and metabolic syndrome in adults. Journal of the American College of Nutrition. 2018; 37(6):487-493.

25. Harpaz D, Yeo LP, Cecchini F, et al. Measuring Artificial Sweeteners Toxicity Using a Bioluminescent Bacterial Panel. Molecules. 2018;23(10):2454.

26. Ruiz-Ojeda FJ, Plaza-Díaz J, Sáez-Lara MJ, Gil A. Effects of Sweeteners on the Gut Microbiota: A Review of Experimental Studies and Clinical Trials. Advances in Nutrition. 2019;10(suppl_1):S31-S48.

27. Bian X, Chi L, Gao B, Tu P, Ru H, Lu K. Gut Microbiome Response to Sucralose and Its Potential Role in Inducing Liver Inflammation in Mice. Front Physiol. 2017;8:487. 28. Rodriguez-Palacios A, Harding A, Menghini P, et al. The Artificial Sweetener Splenda Promotes Gut Proteobacteria, Dysbiosis, and Myeloperoxidase Reactivity in Crohn's Disease-Like lleitis. Inflammatory bowel diseases. 2018;24(5):1005-1020.

29. Rodriguez-Palacios A, Cominelli F. Myeloperoxidases and Proteobacteria: Reliable Interspecies Biomarkers to Identify and Monitor Pro-inflammatory Diets in Humans. Inflammatory bowel diseases. 2019;25(2):e1-e2.

30. Gerasimidis K, Bryden K, Chen X, et al. The impact of food additives, artificial sweeteners and domestic hygiene products on the human gut microbiome and its fibre fermentation capacity. European Journal of Nutrition. 2019; 1-18.

31. Lobach AR, Roberts A, Rowland IR. Assessing the in vivo data on low/no-calorie sweeteners and the gut microbiota. Food Chem Toxicol. 2019;124:385-399.

32. Plaza-Diaz J, Pastor-Villaescusa B, Rueda-Robles A, Abadia-Molina F, Ruiz-Ojeda FJ. Plausible Biological Interactions of Low- and Non-Calorie Sweeteners with the Intestinal Microbiota: An Update of Recent Studies. Nutrients. 2020;12(4):1153.

33. Liu CW, Chi L, Tu P, Xue J, Ru H, Lu K. Quantitative proteomics reveals systematic dysregulations of liver protein metabolism in sucralose-treated mice. J Proteomics. 2019;196:1-10.

34. Pham HT, Stevens JE, Rigda RS, et al. Effects of intraduodenal administration of the artificial sweetener sucralose on blood pressure and superior mesenteric artery blood flow in healthy older subjects. Am J Clin Nutr. 2018;108(1):156-162.

35. Dai X, Guo Z, Chen D, et al. Maternal sucralose intake alters gut microbiota of offspring and exacerbates hepatic steatosis in adulthood. Gut Microbes. 2020;11(4):1043-1063.

36. Azad MB, Archibald A, Tomczyk MM, et al. Nonnutritive sweetener consumption during pregnancy, adiposity, and adipocyte differentiation in offspring: evidence from humans, mice, and cells. Int J Obes (Lond). 2020;10.1038/s41366-020-0575-X.

37. Rother KI, Sylvetsky AC, Walter PJ, Garraffo HM, Fields DA. Pharmacokinetics of Sucralose and Acesulfame-Potassium in Breast Milk Following Ingestion of Diet Soda. J Pediatr Gastroenterol Nutr. 2018;66(3):466-470.

38. Rother KI, Sylvetsky AC, Schiffman SS. Non-nutritive sweeteners in breast milk: perspective on potential implications of recent findings. Arch Toxicol. 2015;89(11):21692171.

39. Sylvetsky AC, Bauman V, Blau JE, et. al. Plasma concentrations of sucralose in children and adults. Toxicol Environ Chem. 2017;99(3):535-542.

40. Garavaglia MB, Rodríguez García V, Zapata ME, et al. Non-nutritive sweeteners: children and adolescent consumption and food sources. Arch Argent Pediatr. 2018;116(3):186-191.
41. Martyn DM, Nugent AP, McNulty BA, et al. Dietary intake of four artificial sweeteners by Irish pre-school children. Food Addit Contam Part A Chem Anal Control Expo Risk Assess. 2016;33(4):592-602.

42. Martínez X, Zapata Y, Pinto V, et al. Intake of Non-Nutritive Sweeteners in Chilean Children after Enforcement of a New Food Labeling Law that Regulates Added Sugar Content in Processed Foods. Nutrients. 2020;12(6):1594.

43. Dewinter L, Casteels K, Corthouts K, et al. Dietary intake of non-nutritive sweeteners in type 1 diabetes mellitus children. Food Addit Contam Part A Chem Anal Control Expo Risk Assess. 2016;33(1):19-26.

44. O'Sullivan AJ, Pigat S, O'Mahony C, Gibney MJ, McKevitt Al. Longitudinal modelling of the exposure of young UK patients with PKU to acesulfame $K$ and sucralose. Food Addit Contam Part A Chem Anal Control Expo Risk Assess. 2017;34(11):1863-1874. 45. O'Sullivan AJ, Pigat S, O'Mahony C, Gibney MJ, McKevitt Al. Probabilistic modelling to assess exposure to three artificial sweeteners of young Irish patients aged 1-3 years with PKU and CMPA. Food Addit Contam Part A Chem Anal Control Expo Risk Assess. 2016;33(11):1660-1671.

46. Bornemann V, Werness SC, Buslinger L, Schiffman SS. Intestinal Metabolism and Bioaccumulation of Sucralose In Adipose Tissue In The Rat. J Toxicol Environ Health A. 2018;81(18):913-923.

47. Pasqualli T, E Chaves PE, da Veiga Pereira L, Adílio Serpa É, de Oliveira LFS, Machado MM. Sucralose causes non-selective CD4 and CD8 lymphotoxicity via probable regulation of the MAPK8/APTX/EID1 genes: An in vitro/in silico study. Clin Exp Pharmacol Physiol. 2020;47(10):1751-1757.

48. Li X, Liu Y, Wang Y, et al. Sucralose Promotes Colitis-Associated Colorectal Cancer Risk in a Murine Model Along With Changes in Microbiota. Front Oncol. 2020;10:710. 49. Sylvetsky AC, Brown RJ, Blau JE, Walter M, Rother KI. Hormonal responses to non-nutritive sweeteners in water and diet soda. Nutr Metab (Lond). 2016;13:71. 50. Martyn D, Darch M, Roberts A, et al. Low-/No-Calorie Sweeteners: A Review of Global Intakes. Nutrients. 2018;10(3):357. 